\title{
The Adventures of the Rocketeer: Accelerated Motion Under the Influence of Expanding Space*
}

\author{
Juliana Kwan $^{\mathrm{A}, \mathrm{D}}$, Geraint F. Lewis ${ }^{\mathrm{A}}$, and J. Berian James ${ }^{\mathrm{B}, \mathrm{C}}$ \\ A Sydney Institute for Astronomy, School of Physics, A28, The University of Sydney, NSW 2006 \\ B Institute for Astronomy, Royal Observatory, Edinburgh EH9 3HJ, UK \\ ${ }^{\mathrm{C}}$ Dark Cosmology Centre, Niels Bohr Institute, University of Copenhagen, \\ Juliane Maries Vej 30, 2100 Copenhagen, Denmark \\ D Corresponding author. Email: jkwan@ physics.usyd.edu.au
}

Received 2009 July 31, accepted 2009 September 8

\begin{abstract}
It is well known that interstellar travel is bounded by the finite speed of light, but on very large scales any rocketeer would also need to consider the influence of cosmological expansion on their journey. This paper examines accelerated journeys within the framework of Friedmann-Lemaitre-Robertson-Walker universes, illustrating how the duration of a fixed acceleration sharply divides exploration over interstellar and intergalactic distances. Furthermore, we show how the universal expansion increases the difficulty of intergalactic navigation, with small uncertainties in cosmological parameters resulting in significantly large deviations. This paper also shows that, contrary to simplistic ideas, the motion of any rocketeer is indistinguishable from Newtonian gravity if the acceleration is kept small.
\end{abstract}

Keyword: cosmology: theory

\section{Introduction}

The discovery of the accelerated expansion of the universe (Riess et al. 1998; Perlmutter et al. 1999) poses an interesting challenge to future space travellers. In the currently favoured cosmological model, the future of intergalactic travel appears grim as the universe becomes dominated by dark energy and interesting structures, such as galaxies and quasars, will have receded beyond the maximum distance that the rocketeer can travel to, even after an infinite proper time. The 'end of cosmology' will be realised as the rocketeer infers the existence of an 'island universe', being able to reach fewer and fewer destinations as these are accelerated beyond their event horizon (Loeb 2002; Krauss \& Scherrer 2007).

In this paper, we consider the path of a rocketeer, who intends to travel as far as possible in a FriedmannLemaître-Robertson-Walker (FLRW) universe with different cosmologies. The nature of the path depends on several important features of the cosmology and this is treated in terms of the limiting horizons alluded to by Krauss \& Scherrer (2007) and Loeb (2002), and also in the use of phase portraits to describe the motion of the rocketeer. We also address some of the problematic aspects of travelling in an expanding universe, including the difficulty of achieving a return trip due to the complicated dependence of the motion of the rocketeer

\footnotetext{
* Research undertaken as part of the Commonwealth Cosmology Initiative (CCI: www . thecci . org), an international collaboration supported by the Australian Research Council.
}

upon the evolution of the energy densities. Furthermore, it is shown that although far more realistic than constant acceleration, an initial burst of acceleration followed by a 'coasting' period is of little use if we wish to traverse intergalactic distances. We also contribute to the current debate of whether space really expands (see Lewis et al. (2008) and Abramowicz (2008) for recent developments) in the context of accelerated motion.

Several authors have explored unaccelerated paths through a FLRW universe. See, for example, Whiting (2004) and Grøn \& Elgarøy (2007) for the general solution for free particle motion in FLRW cosmologies as well as Barnes et al. (2006) who clarify the interpretation of these solutions in the context of joining the Hubble flow. However, the treatment of accelerated motion is uncommon, despite its potential for illuminating the nature of expanding space as a physical phenomenon or a trick of coordinates. Including the effect of acceleration increases the complexity of the problem and indeed have only been discussed at length in the context of cosmology by Rindler (1960) and Heyl (2005). Rindler (1960) presented a novel method for calculating accelerated paths by reducing the order of the differential equations albeit by increasing the number of coupled equations to solve. Of more relevence, Heyl (2005) considered the problem of how far a rocketeer can travel in a human lifespan.

The layout of this paper is as follows: Section 2 presents the necessary background including the equations of motion of the rocketeer, while Section 3 addresses the return journey of a rocketeer in a FLRW universe and its 
implications for the concept of expanding space; Section 4 discusses the journey of an intergalactic explorer who initially accelerates but then 'coasts' at a constant velocity close to the speed of light. Furthermore we also discuss the importance of accelerated motion in the context of future measurements in extragalactic astronomy. In the Appendix, we provide a first-order perturbative solution for the motion of the rocketeer.

\section{Background}

Accelerated motion in general relativity is most conveniently approached by solving the geodesic equation with a four-acceleration term. The equation of motion of the rocketeer is given by:

$$
\epsilon^{\mu} \equiv \frac{\mathrm{D} u^{\mu}}{\mathrm{d} \tau}=\frac{\mathrm{d} u^{\mu}}{\mathrm{d} \tau}+\Gamma_{\nu \sigma}^{\mu} \frac{\mathrm{d} x^{\nu}}{\mathrm{d} \tau} \frac{\mathrm{d} x^{\sigma}}{\mathrm{d} \tau},
$$

where $\epsilon^{\mu}$ is the four acceleration, $u^{\mu}$ is the four-velocity and $\tau$ is the proper time of the rocketeer. The magnitude of the acceleration, $\epsilon$, corresponds to the force experienced by the rocketeer. Equation 1 constitutes a set of differential equations that are generally coupled for non-trivial systems once an appropriate geometry has been inserted. Solving these is the first step in determining the path of the rocketeer, but for many cases this is analytically intractable and we have had to recourse to numerical techniques extensively. We present a pertubative solution (whose accuracy is limited to specific regimes of proper time) in the Appendix, but this is unwieldy and cumbersome. It is also necessary to choose an appropriate means of slicing the time coordinate for the FLRW model; in this case it is most expedient to work in conformal coordinates. The path of the rocketeer asymptotes to a null geodesic, as shown in Heyl (2005), which determines the maximum comoving distance that can be travelled in an infinite proper time. This is easily visualised when all photon paths trace out straight lines oriented at $\pm 45^{\circ}$, as in special relativity and although this is not true in general for curved spacetimes, we can enforce this behaviour through a conformal representation. Note that such a transformation preserves angles at a point and as such paths that are timelike in FLRW coordinates remain so in the conformal geometry and vice versa. Since we are only considering motion in a radial direction, we can express the FLRW metric:

$$
\mathrm{d} s^{2}=a^{2}(\eta)\left(\mathrm{d} r^{2}-\mathrm{d} \eta^{2}\right)
$$

where $\eta$ is the conformal time and $a(\eta)$ is the scale factor that governs the expansion of the universe. The conformal time is simply a rescaling of the coordinate time (the time measured by a stationary observer in a FLRW metric), using:

$$
a(t) \mathrm{d} \eta \equiv \mathrm{d} t .
$$

Equation 2 is only valid if we restrict our discussion to two dimensions or universes without spatial curvature; if we wish to consider a more general treatment, then it is necessary to perform a transformation to fully conformal coordinates, in which the spatial coordinates must be modified as well (see Infeld \& Schild (1945) and Lewis et al. (2007) for a complete derivation).

Simply solving Equation 1 is not sufficient to derive the motion of the rocketeer; she must also satisfy the following constraints from the normalisation of $u^{\mu}$, the orthogonality of $u^{\mu}$ and $\epsilon^{\mu}$ and the magnitude of $\epsilon^{\mu}$ (Equations 4, 5 and $6)$ that are inserted into their initial conditions. These are expressed in the FLRW metric, as follows:

$$
\begin{aligned}
& g_{\mu \nu} u^{\mu} u^{\nu}=a(\eta)^{2}\left(u^{r^{2}}-u^{\eta^{2}}\right)=-1, \\
& g_{\mu \nu} \epsilon^{\mu} \epsilon^{\nu}=a(\eta)^{2}\left(\epsilon^{r^{2}}-\epsilon^{t^{2}}\right)=\epsilon^{2}, \\
& g_{\mu \nu} u^{\mu} \epsilon^{\nu}=u^{r} \epsilon^{r}-u^{t} \epsilon^{t}=0,
\end{aligned}
$$

where the rocketeer is restricted to travel in the radial direction only. Since this scenario is purely intended to address the issue of inertial observers in expanding space, we have ignored the details on how such a journey might be technically feasible and the rocketeer is represented by a point particle of unspecified mass that is capable of sustaining a constant acceleration for an indefinite period.

\section{Accelerated Motion}

To begin, we would like to consider the simplest case of accelerated motion in an expanding universe: constant acceleration in a single direction. For a universe described purely by special relativity, it is trivial to show that the path of a rocketeer is a hyperbola of the form:

$$
t=\frac{1}{\epsilon} \sinh (\epsilon \tau) ; \quad r=\frac{1}{\epsilon} \cosh (\epsilon \tau)
$$

In this instance, constant acceleration means that the threevelocity of the rocketeer rapidly approaches the speed of light and her path is well approximated by that of a photon as $\eta=r+1 / \epsilon$, where $r$ is the comoving radial coordinate.

A similar analysis may be performed in conformal coordinates to determine how far the rocketeer can travel after an infinite time has elasped because her path is bounded by a light cone projected from her point of departure. For universes with a non-zero cosmological constant, the conformal time in the future is finite after an infinite cosmic time has elapsed ${ }^{1}$. Heyl (2005) showed that the furthest the rocketeer can travel is a comoving distance equal to the remaining conformal time for that cosmological model, which corresponds to travelling for an infinite cosmic time; the transformation is purely for convenience. As cosmic time approaches infinity, a universe with a non-zero cosmological constant will have a scale factor that is well approximated by an exponential (and is exact for $\Omega_{\Lambda, 0}=1$ and $\Omega_{\mathrm{m}, 0}=0$ ). Thus, the conformal time remains finite in all of these models, because the integral that defines conformal time (Equation 3) will always converge. However, the rocketeer only reaches the maximum distance after an infinite proper time has elapsed, but she is able to

\footnotetext{
${ }^{1}$ In fact, any cosmology dominated by a fluid with equation of state parameter less than $-1 / 3$ contains a finite conformal future.
} 


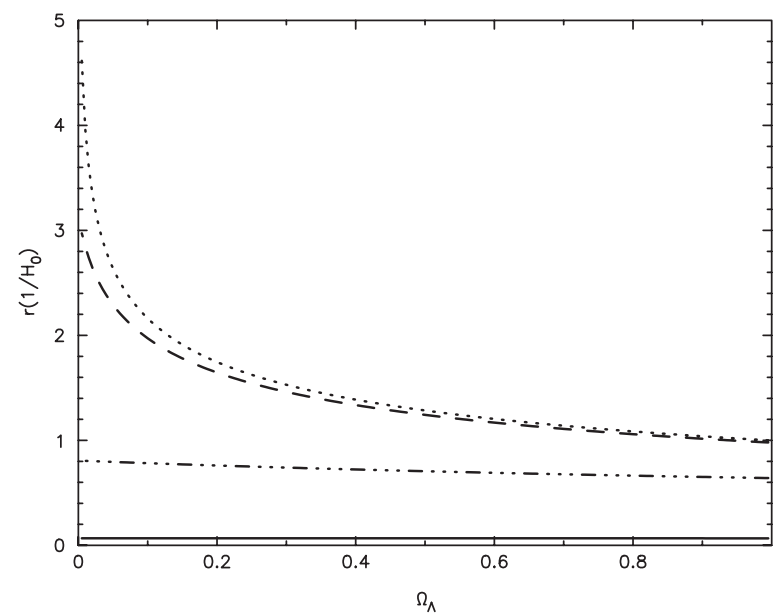

Figure 1 Maximum radial comoving distances travelled by a rocketeer with $\epsilon=g$ for proper times of 20 (solid), 23 (dot-dashed), 26 (dashed) and 29 (dotted) years. The rocketeer covers most of her journey between $\approx 23$ and 26 years as measured in her frame. Indeed, after 26 years, the rocketeer hardly travels any further in cosmologies with a non-zero $\Lambda$. From this point of view, a non-zero cosmological constant is actually advantageous, since for large values of $\Omega_{\Lambda}$, it takes very little time to reach a substantial portion of the maximum distance accessible to an intergalactic explorer. We have assumed $\Omega_{\Lambda}+\Omega_{\mathrm{m}}=1.0$ and that each journey starts from the current time in each universe.

travel a comoving distance of $\approx 4.63 \mathrm{Gpc}$ (99\% of the maximum conformal time, $\eta=1.12339$ ) in $\approx 27$ years in the currently favoured concordance model with parameters $\Omega_{\mathrm{m}, 0}=0.27, \Omega_{\Lambda, 0}=0.73$ and $H_{0} \approx 72 \mathrm{~km} \mathrm{~s}^{-1} \mathrm{Mpc}^{-1}$ if she leaves now. Note that for this model, Heyl (2005) calculates a different value for the proper time taken to traverse the same comoving distance and has his rocketeer accelerating for $\approx 50$ years of proper time instead to cover 99\% of the maximum distance with the same acceleration and cosmology.

In Figure 1, we have calculated how far the rocketeer reaches after travelling for various proper times at an acceleration of $\epsilon=g=9.81 \mathrm{~m} \mathrm{~s}^{-2}$. As in Heyl (2005), we find that the majority of the maximum distance is covered between $\approx 20$ and 23 years, except when $\Omega_{\Lambda} \approx 0$. Thus, for short trips, the greatest distance reached is largely unaffected by the amount of dark energy in the universe. Up to $\approx 23$ years, the total distance travelled is well described by a straight line with a very small gradient over the range of energy densities, which implies that the behaviour of the scale factor, for all spatially flat FLRW models with a cosmological constant, is mostly irrelevant for the distances covered by the rocketeer. It is tempting to think that it is possible to just approximate the full solution with special relativity up to a significant distance but the discrepancy is actually quite large in comparison to the numerical result (see the Appendix for a detailed discussion).

But suppose that a rocketeer wished to travel to a distant galaxy with the intention of returning home; how would she have to fire her rocket to bring her to her destination and back again in an expanding universe? A single return journey as shown on the left panels in Figure 2 is modelled

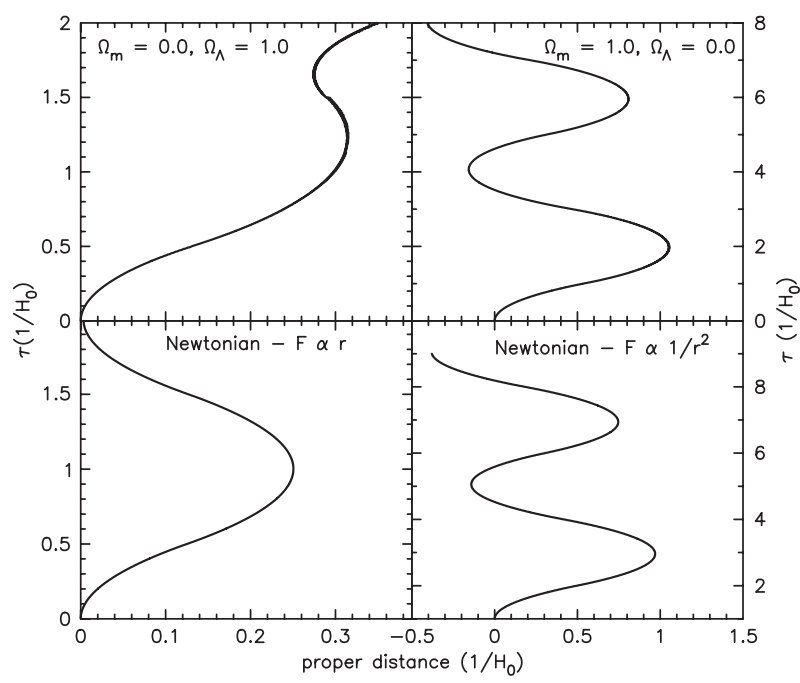

Figure 2 Return paths of rocketeers in various cosmological models. A single return journey consists of three stages: starting at $\tau=0$, the rocketeer accelerates outwards at $\epsilon=H_{0}$ until $\tau=H_{0}^{-1}$, decelerates at $\epsilon=-H_{0}$ until $\tau=3 H_{0}^{-1}$, and finally accelerates at $\epsilon=H_{0}$ until $\tau=4 H_{0}^{-1}$ to attempt to return to the origin and stop at rest relative to the cosmological fluid. All radial distances are measured in proper distances.

by applying an acceleration that is a piecewise function of proper time, such that:

$$
\epsilon=\left\{\begin{aligned}
1 & \text { if } 0 \leq \tau<1 / H_{0} \text { or } 3 / H_{0} \leq \tau<4 / H_{0} \\
-1 & \text { if } 1 / H_{0} \leq \tau<3 / H_{0}
\end{aligned}\right.
$$

and this is repeated to produce the two return paths on the right. We have used fairly long travel times and correspondingly very small accelerations $\left(\epsilon=H_{0} \approx 6.85 \times 10^{-11} \mathrm{~g}\right.$ ) to exaggerate any effects that the evolution of the scale factor may have on the path of the rocketeer, since for large accelerations, the trajectory asymptotes to a straight line soon after blast off. Three universes have been chosen for their interesting properties; Figure 2 shows the path of a rocketeer in a de Sitter model $\left(\Omega_{\mathrm{m}, 0}=0, \Omega_{\Lambda, 0}=1\right)$ and an Einstein-de Sitter model $\left(\Omega_{\mathrm{m}, 0}=1, \Omega_{\Lambda, 0}=0\right)$ in the top two panels. We have also shown the path of the rocketeer under Newtonian gravity (see Tipler 1996; Whiting 2004) in the other two panels. The bottom right panel of Figure 2 uses the familiar $1 / r^{2}$ force law, but Newtonian gravity also admits a lesser known linear force law for which Gauss's law still holds (Calder \& Lahav 2008). The inclusion of this term renders the Newtonian force law into a form directly comparable to the Friedmann solution with a cosmological constant in the limit of weak gravitational fields as the coefficient of the term in $r$ behaves like $\Lambda$. Thus, we can mimic a de Sitter model using an acceleration $\propto r$ and an Einstein-de Sitter universe with an acceleration $\propto 1 / r^{2}$, the results of which are analysed along the bottom row of Figure 2. Although, it is difficult to discern in the left hand panels, each path in a GR-Newtonian pair has the same frequency with respect to proper time. Additionally, we analyse the motion of a rocketeer in a Milne or empty 
universe $\left(\Omega_{\mathrm{m}, 0}=\Omega_{\Lambda, 0}=0\right)$ under the same type of return journey in Figure 3 for comparison, since in this scenario, the spacetime is clearly static, as discussed below.

The top row of Figure 2 shows that in a FLRW universes, the rocketeer does not return to the origin on her way back home if her accelerations are symmetric with respect to her proper time. We could choose to view this as an effect of 'expanding space'; space grows bigger as she travels and so the rocketeer is being carried away as if on a rubber sheet as the universe expands. Where the analogy fails is for the matter-dominated case: we would expect that as the balloon is blown up, albeit at a decreasing rate, the distance travelled is greater on the outbound leg than on the way back. The crucial difference, of course, is the absence of gravity which causes the rocketeer to overshoot in a matter dominated universe and undershoot in a de Sitter universe. For motion restricted to the radial direction, the relationship for the change in rapidity, $\chi=\tanh ^{-1}\left(u^{r} / u^{t}\right)$, with proper time is given by:

$$
\frac{\mathrm{d} \chi}{\mathrm{d} \tau}=\epsilon-\frac{\dot{a}(\eta)}{a^{2}(\eta)} \sinh \chi,
$$

(see Heyl (2005) for further details). Heyl (2005) describes the second term in Equation 9 as a 'friction term' that results from the expansion of the universe. In fact, $H(\eta)=\dot{a}(\eta) / a^{2}(\eta)$, so we can view the significance of this term in the above equation as a Hubble drag term that limits the increase in the rapidity of the rocket.

The Hubble parameter describes the expansion history of the universe and is given by:

$$
H=H_{0} \sqrt{\Omega_{\mathrm{m}, 0} a^{-3}+\Omega_{\Lambda, 0}},
$$

for spatially flat FLRW models. We can immediately see that this is going to be greater for accelerating universes than decelerating ones at the same point in cosmic time. For instance, the Hubble parameter of an universe with a mixture of matter and dark energy asymptotes to the de Sitter case as $t \rightarrow \infty$, so the maximum amount of 'drag' is always obtained for the rocketeer in a dark energy dominated universe. For an empty universe, the second term on the right hand side of Equation 9 is zero. Thus the rocketeer in a matter dominated universe where $\dot{a}(\eta) / a^{2}(\eta)$ is decreasing, reaches a higher rapidity in the same proper time as a rocketeer in the concordance model with accelerated expansion and overshoots. However, the physics behind the analogy is simply that the influence of changing energy densities on the path of the rocketeer cannot be ignored (Lewis et al. 2008). Recently, there has been much confusion in the literature regarding the physicality of expanding space. It had been suggested by some (Abramowicz et al. 2007, 2008; Abramowicz 2008) that the expansion of space is a physical phenomenon with observable consequences rather a byproduct of a choice of coordinates. The above asymmetry is apparently one of the reasons why Abramowicz et al. (2007), Abramowicz (2008) and Abramowicz et al. (2008) support the notion of expanding space as a measurable effect: the motion of particles in a FLRW universe is not purely described by special relativity but is distorted by space time curvature. In fact, the behaviour of a rocketeer in Newtonian gravity is very similar to a rocketeer in a matter-dominated universe. Previously, it has been shown that, up to $\approx 100 \mathrm{Mpc}$, geodesic motion is qualitatively well approximated by Newtonian gravity (Barnes et al. 2006). With an accelerated observer, however, we cannot make a similar universal statement to a give a scale on which Newtonian gravity is an accurate approximation, because this is of course dependent on the rocketeer's acceleration and the cosmology; this is evident from comparing the left and right panels of Figure 2. But as this figure also demonstrates, both rocketeers overshoot; it is misleading to attribute this behaviour in an Einstein-de Sitter model to the stretching of space carrying away the rocketeer. It is sufficient to say that the changing effect of gravity during the rocketeer's journey, distorts her path such that the outward leg of her trip is not the same as the inbound leg without having to invoke the analogy of an expanding balloon.

In contrast, the behaviour of a rocketeer using a de Sitter model and a linear Newtonian force in $r$ are only similar up to a proper time of $\approx 0.8 H_{0}^{-1}$ after which the paths diverge. It is easier to interpret this situation as an example of expanding space in action, whereby the rocketeer is being dragged away by the rapid increase in the scale factor. But the rocketeer can only travel for as long as the remaining conformal time in the de Sitter case, which can not be mimicked in a Newtonian sense. The conformal factor $1 / a(t)$ becomes negligible as $\eta \rightarrow H_{0}^{-1}$ and at this time, the Newtonian approximation breaks away from the GR picture. Again we are presented with two choices for interpreting this situation; either the exponential increase in the scale factor causes the rocketeer to cover a greater proper distance with each trip or that $u^{r}$ increases as the cosmic time approaches infinity to preserve the normalisation of the four-velocity as $u^{\eta} \rightarrow 0$. Each interpretation is only separated by the choice of a metric, again we reiterate that expanding space is a coordinate dependant effect as emphasised in Lewis et al. (2008) and Bunn \& Hogg (2008). Furthermore, a careful inspection of the Newtonian case reveals that the rocketeer really did undershoot after all, although the amount by which this occurs is still an order of magnitude away from its behaviour in GR.

Figure 3 corresponds to an universe empty of all gravitating material in which the rocketeer is able to return to the origin at rest in the same amount of proper time as the outward journey. Although its scale factor is given by $a(t)=H_{0} t$, this is a static model developed from special relativity Milne (1932) to describe a universe consisting entirely of non-gravitating particles, since it is possible to relate the FLRW metric of the empty universe as a function of the comoving radial coordinate, $r$, and the cosmic time $t$, to the metric of special relativity with coordinates $R, T$ via the following transformation (Grøn 2006; Rindler 2006):

$$
R=t \sinh r, \quad T=t \cosh r
$$




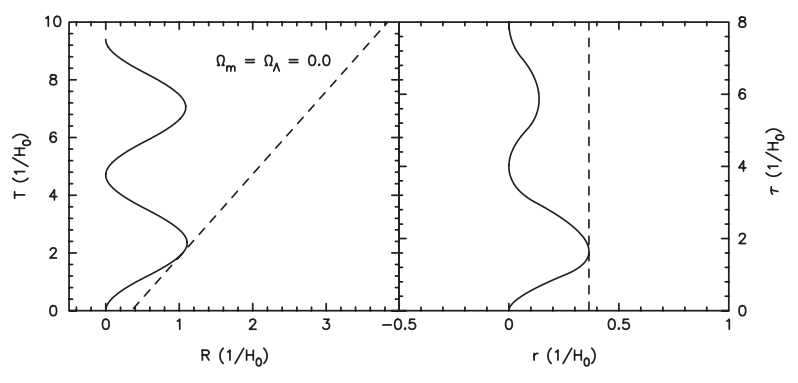

Figure 3 Return paths in the Milne model or empty universe. Her journey is structured in the same way as in Figure 2. The above plots were produced by repeating a return trip twice and we have also included a comoving observer in dashed lines for comparison. The rocketeer meets the comoving observer only once during her journey and hence the path of a comoving observer in fully comoving coordinates $(R, T)$ is tilted. The left panel shows her journey in conformal coordinates $(R, T)$, while the path on the right is in terms of comoving coordinates.

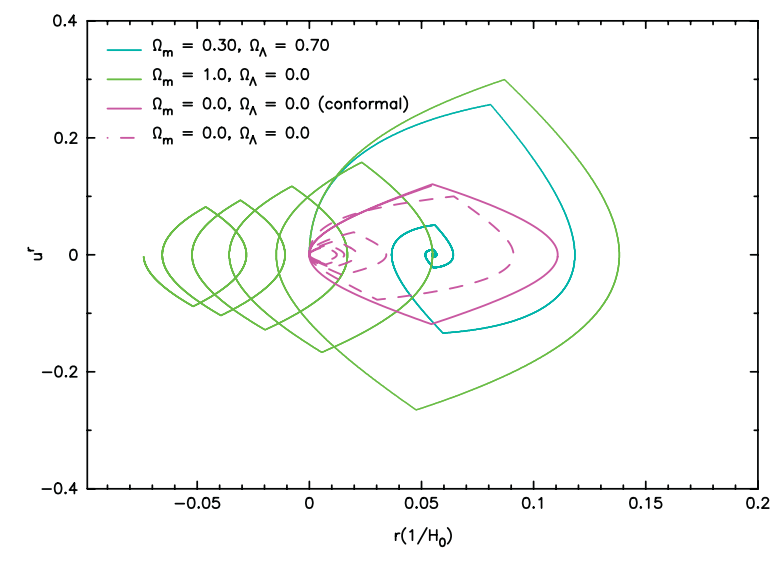

Figure 4 Phase portraits for three different FLRW models. The paths show five consecutive return journeys under the same conditions as in Figures 2 and 3 for three cosmologies, except the duration of each trip has been halved. Each universe has a characteristic phase profile according to its long term behaviour. The Milne model has been plotted in both synchronous and conformal coordinates; as well as being scaled down to $10 \%$ (solid) and $25 \%$ (dashed) of its original amplitude.

such that the line element becomes $\mathrm{d} S^{2}=-\mathrm{d} T^{2}+\mathrm{d} R^{2}$ in two dimensions. Thus we can consider the motion as being characterised by special relativity; surfaces of constant $t$ have negative curvature, but the curvature overall tends to zero as $t$ increases. Both axes of the lower left panel of Figure 3 are plotted in fully conformal coordinates, $R$ and $T$. Since this universe is actually static, each oscillation with respect to $T$ retains the same shape.

The long term behaviour of the rocketeer may be neatly encapsulated in the phase portraits shown in Figure 4. Again, we have chosen three universes with very different cosmic evolutions: the accelerated expansion of the concordance model (cyan), the eternally coasting Einstein-de Sitter universe (green) and the SR metric in the guise of a Milne universe (purple). These paths were produced using the same acceleration scheme as Figures 2 and 3 using Equation 8 but have been extended for five oscillations to provide a more detailed curve. Since the acceleration of a rocketeer is symmetric about the origin for a Milne universe (see Figure 3), her phase portrait is a conservative, closed orbit and she always returns back to the origin. The phase portraits describing the other models are much more informative: the path in the concordance cosmology clearly converges towards a particular point as the rocketeer's journey is bounded by a finite conformal time, while the phase portrait of a rocketeer in an Einstein-de Sitter universe spirals to infinity and indeed would do so if it had not been truncated to five oscillations. Thus the type of orbit cleanly delineates the type of cosmological horizon present in each model. However the point that the rocketeer tends towards in the concordance model is strictly not an attractor, since if we started from a different comoving coordinate, the destination of the rocketeer would also be shifted, but an attractor would exist at $\left(\eta, u^{r}\right)=(1.12339$, 0) for the concordance model.

The dependence on cosmology in the equations of motion introduces additional complexity that makes it unclear if a general solution that can always return the rocketeer to the origin exists. If she is allowed to change only the magnitude of her acceleration on the return leg, the equations are over constrained and it is not possible to return to the origin for any value of $\eta$. However adjusting both its magnitude and duration rectifies this. Figure 5 shows an example of such a scenario in an Einstein-de Sitter universe in which the rocketeer trials several values of $\eta$ and stops when she reaches $u^{r}=0$. The outward journey remains the same as in Figure 2, the red curve corresponds to $\epsilon=1$ for $H_{0}^{-1}$ and the green curve corresponds to $\epsilon=-1$ for $2 H_{0}^{-1}$, but on her final leg we have explored the effect of varying the magnitude of $a$ as well as the length of proper time for which she accelerates. The rocketeer would be able to return to rest at the origin if she accelerated at $\epsilon=1.28639 H_{0}$ for $0.829959 H_{0}^{-1}$. But her success relies heavily on being well informed about the cosmology before she commences her journey, since any observation that she performs would be distorted by relativistic aberration. The black curve in Figure 5 shows an unfortunate traveller who misjudges his cosmology by $0.1 \%$. Such a mistake would result in arriving $\approx 1.18490 \times 10^{-2} H_{0}^{-1} \approx 49.3 \mathrm{Mpc}$ from Earth.

\section{Expanding Space: A Traveller's Guide}

How can we best exploit the expansion of the universe such that future space travellers can explore as much of the universe as they can in a reasonable amount of proper time? Heyl (2005) considered this question for a rocketeer with a constant acceleration, but achieving this for the lifespan of a rocketeer may prove to be rather unrealistic (Rindler 1960). It is appropriate then, to consider the amount by which the path of a rocketeer diverges from that of a 'coaster', a traveller of intermittent acceleration, if at all. Naïvely we might expect that the Hubble drag term would force the motion of the coaster back into the general expansion of the universe, as discussed in Barnes et al. (2006) for geodesic motion. In Figure 6, we have plotted the results from solving for such a situation 

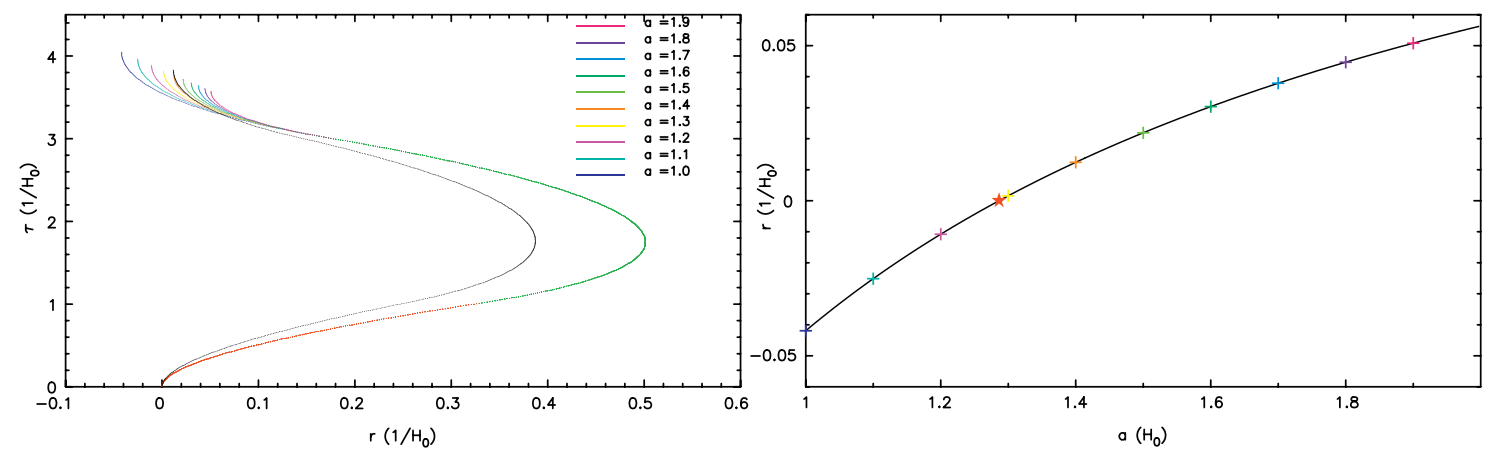

Figure 5 Return paths for a rocketeer who accelerates outwards in the same manner as in Figures 2-4 but adjusts both the magnitude and length of the her acceleration during the final stage of her journey such that $u^{r}=0$ at the end. We have used an Einstein-de Sitter model, which lacks the added complication of a finite conformal time. The most favourable of these paths is marked with a red star in the figure on the right, which shows the final comoving radial coordinate of the rocketeer at $u^{r}=0$ according to her acceleration on her final leg; it appears that the rocketeer is able to return to the origin if she accelerates at $\epsilon=1.421337 H_{0}$ for $0.750610 H_{0}^{-1}$. The black line corresponds to a rocketeer who uses these parameters but in an universe with the cosmological parameters $\Omega_{\mathrm{m}, 0}=0.999, \Omega_{\Lambda, 0}=0.001$. Coloured crosses on the right hand panel correspond to paths on the left with the same colour.

in three different universes with the same parameters as those in Section 3. The path of rocketeer travelling with $\epsilon=10 H_{0}$ is shown as a solid line with a set of rocketeers who accelerate at $\epsilon=10 H_{0}$ initially, but then switch off their engines at $u^{r}=2$ (dotted line), $u^{r}=2.5$ (dot-dashed line) and $u^{r}=3$ (dashed line). The difference in the final comoving coordinates achieved by the coasters and rocketeer is not immediately apparent from Figure 6, since the paths of the rocketeer have been truncated for the middle and bottom panels to maintain a reasonable scale between the three worldlines.

It is apparent from the conformal diagrams on the left of Figure 6, that while the coaster and the rocketeer have similar paths in all the conformal representations, after the coasters turns off their engines, they experience a significantly smaller amount of time dilation when compared to the rocketeer. For a particular comoving radial coordinate in the diagrams on the right, the proper times measured by each vary dramatically and only the coasters in the concordance model are a comparable distance from the rocketeer in comoving coordinates (but are separated by a significant proper distance). While the coasters may keep up with the rocketeer in terms of their comoving spatial separation, the times they would measure would be considerably greater than that on the clock carried by the rocketeer. However, if we take slices at a constant conformal time, both the rocketeer and the coasters in all three universes are at similar radial coordinates, which implies that the coasters skim along the light cone approached by the rocketeer.

With a more realistic acceleration of $\epsilon=g$, a traveller in the concordance model, who starts to coast after reaching a three-velocity of $0.8 c$ at a comoving distance of $0.189 \mathrm{pc}$, can expect to travel $\approx 41.1 \mathrm{pc}$ after a total proper time of 100 years has elapsed. Even with a maximum threevelocity of $0.99 c$, the coaster can only accelerate up to $1.67 \mathrm{pc}$ after a year, and must drift for a further 99 years to reach $\approx 208.5 \mathrm{pc}$. This is much less than the furthest distance travelled by a rocketeer in the same proper time $(\approx 4.7 \mathrm{Gpc})$ and coasting appears to be of little use in intergalactic exploration. It seems that in this scenario, in which the distance travelled by the coaster before switching off her engines is much less than the maximum conformal time, it is not practical for a rocketeer to alter between accelerated and geodesic motion. However, if a spacecraft of limited acceleration is the only means of travel available, a coaster could maximise their final comoving distance by accelerating up to $\approx 26$ years (or $\approx 23$ years for a lower bound). There is a preferred duration for which the rocketeer should accelerate, to maximise the distance that she covers while minimising the amount of proper time spent travelling. Recall from Figure 1 that after this period, the rocketeer adds little to her journey, at least for $\Lambda$-dominated universes. This can be understood from a comparison of the results shown in Figure 1 with the panels on the right in Figure 6; if the coaster switches to non-accelerated motion too early in her journey, she is not experiencing the rapid jump in the comoving distance travelled by the rocketeer occurring between $20 \lesssim \tau \lesssim 26$ years (in Figure 1) and $0.3 \lesssim \tau \lesssim 0.4 H_{0}^{-1}$ (in Figure 6). However, this is only applicable to universes containing with a finite conformal time; a coaster in an Einstein-de Sitter universe will always be much more disadvantaged than a rocketeer.

Recently, Krauss \& Scherrer (2007) have concluded that as the behaviour of our Universe becomes increasingly dominated by dark energy, future astronomers will infer the existence of a static universe as described by the de Sitter metric, written its original form:

$$
\mathrm{d} s^{2}=\left(1-\frac{r^{2}}{R^{2}}\right) \mathrm{d} t^{2}-\frac{\mathrm{d} r^{2}}{1-r^{2} / R^{2}}-r^{2} \mathrm{~d} \Omega^{2},
$$

where $R$ is the radius of a 4D hypersphere in 5D Euclidean space (de Sitter 1917). While a coordinate transformation links this metric to the synchronous gauge, that it can be written in terms of a cosmological constant will be nothing more than a mathematical curiosity as cosmic acceleration is unlikely to be supported by observational evidence in the very distant future. All objects not gravitationally bound to our Galaxy will have receded such that neither the Hubble expansion or the elemental abundances in quasar 

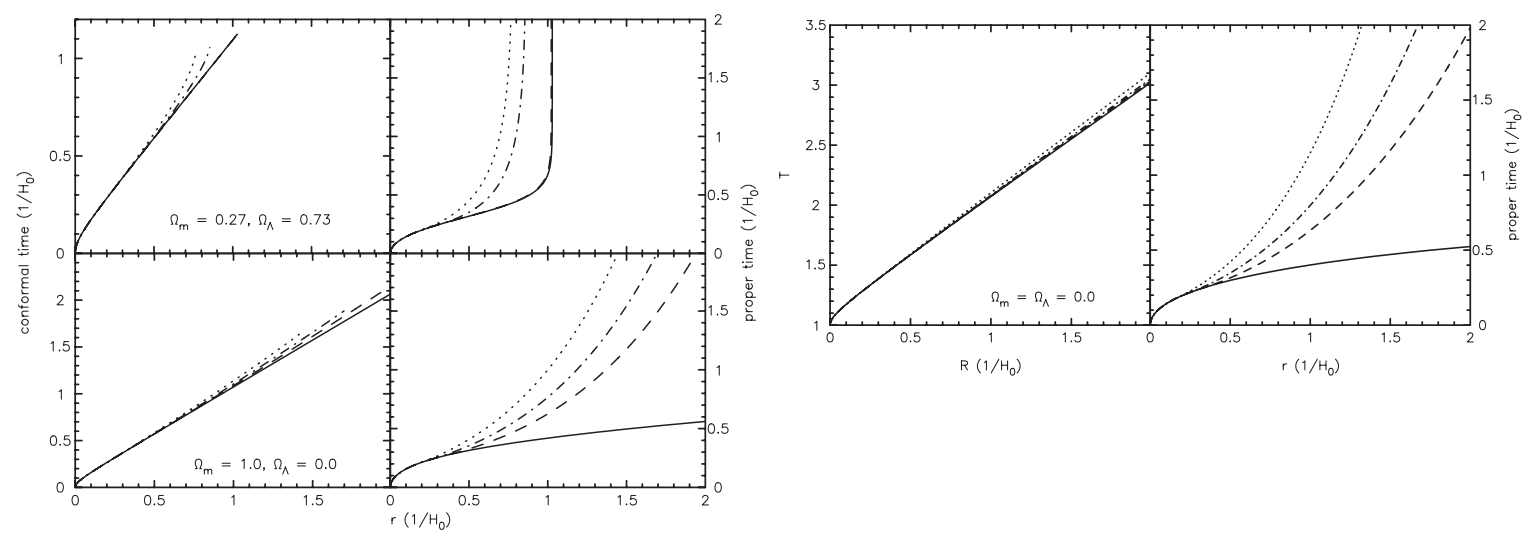

Figure 6 Rocketeer vs. coaster for different universes, with their cosmological parameters shown on the bottom right. We have plotted the paths of several rocketeers with a constant outward acceleration of $\epsilon=10 H_{0}$ who then switch off their engines when $u^{r}=2$ (dotted line), $u^{r}=2.5$ (dot-dashed line) and $u^{r}=3$ (dashed line). The solid line gives the path for a rocketeer who accelerates outwards at $\epsilon=10 H_{0}$ for the entire journey. A comparable amount of total proper time passes for the rocketeers in each universe. As in Figure 2, worldlines in the empty universe have been plotted in fully conformal coordinates using Equation 11. Note that the axis has been truncated at $R=10 H_{0}^{-1}$ and $T=10 H_{0}^{-1}$ for clarity. Notice that the coaster in dashed lines $\left(u_{\max }^{r}=3\right)$ separates at a later time in the concordance model than in any other universe because it takes longer to reach the same velocity with cosmic acceleration.

spectra will be discernible and the intensity of the CMB will have been redshifted below our observational capacity. While an observer stationed on Earth will not detect the presence of dark energy, a rocketeer travelling beyond the Local Group could provide a simple means of determining the correct cosmological parameters. In Section 3, it was shown that with constant accelerated motion, cosmological distances can be travelled well within a human lifespan. Although the conformal time bounds how far the rocketeer can travel such that she may not be able to reach a sufficiently distant destination to observe any interesting structure, the most useful experiment that she could carry out would be to travel in a return path with a constant acceleration or act as a coaster. As demonstrated in the preceding section, the path of rocketeer is extremely sensitive to the amount of dark energy contained in the universe and in fact, there is quite a significant deviation between two paths with cosmologies that differ by only $0.1 \%$ (see previous section). However, while this experiment will only take a few years for the rocketeer, cosmologists on Earth will have to wait a very long time for their results.

\section{Conclusions}

Accelerated motion provides a means for exploring a significant fraction of our universe within the remaining conformal time in a reasonably short time. In this work, we have examined solutions of the equation of motion corresponding to accelerated round-trips in a spatially flat Universe. It has been demonstrated that these paths through spacetime are marked by the action of cosmic evolution, and the manner in which cosmological parameters constrain the properties of the acceleration for the purpose of completing the return trip has been investigated. Moreover, accelerated paths illustrate the pedagogical features of conformal diagrams: this is evident in the characterisation of curves in the phase portraits and also in the

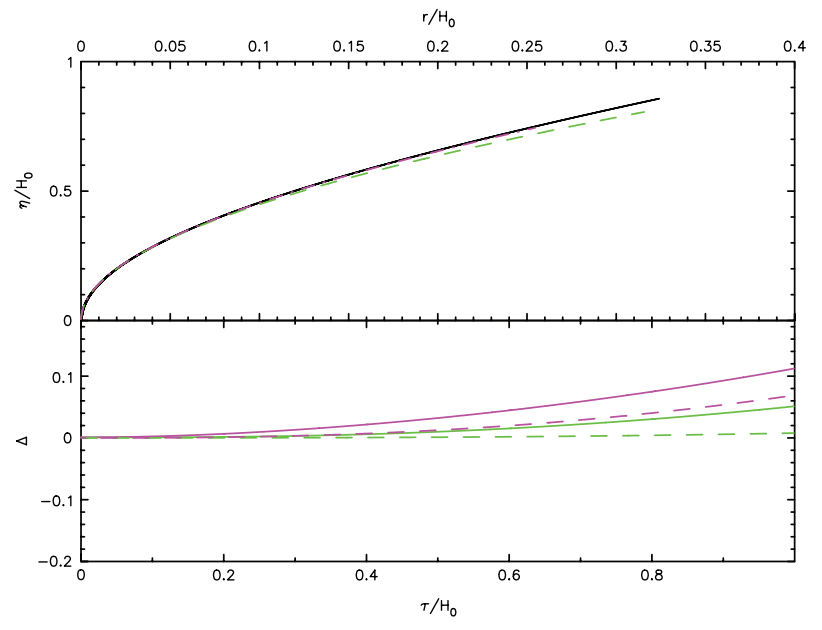

Figure 7 A comparison between various solutions for accelerated paths through an Einstein-de Sitter model $\left(\Omega_{\mathrm{m}, 0}=1, \Omega_{\Lambda, 0}=0\right.$, $k=0$ ), with $\epsilon=1$. In the top panel, we have shown the paths that are obtained from numerically integrating Equation 1 (solid black), the first order solution of this equation in conformal coordinates (Equations 20 and 21, green dashed) and the solution in special relativity (Equation 7, pink dot-dashed). The bottom panel shows the error, $\Delta$, between the numerical result and the solution from perturbation theory and the error between the numerical result and the solution in special relativity for both the conformal time (solid lines) and the radial distance (dashed lines) of the rocketeer. The colours have been chosen to match those of the top panel.

failure of the rocketeer to return exactly the origin. Using these results, we have discussed the capacity for accelerated paths to cover large distances across the Universe. By comparing different rates of acceleration, we have demonstrated that particular choices of motion can be chosen to optimise particular scientific outcomes. That is, while coasting through a FLRW universe by using an intermittent acceleration is an ineffective way of exploring our Universe, accelerated motion may provide the only means of determining a correct description. 


\section{Acknowledgments}

The authors wish to acknowledge support from ARC Discover Project DP0665574 and Matt Francis for his comments and suggestions.

\section{References}

Abramowicz, M. A., Bajtlik, S., Lasota, J.-P. \& Moudens, A., 2007, AcA, 51, 139

Abramowicz, M. A., 2008, NewAR, 51, 799

Abramowicz, M. A., Bajtlik, S., Lasota, J.-P. \& Moudens, A., 2009, AcA, 59, 131

Barnes, L. A., Francis, M. J., James, J. B. \& Lewis, G. F., 2006, MNRAS, 373, 382

Bender, C. M. \& Orszag, S. A., 1978, Advanced Mathematical Methods for Scientists and Engineers: Asymptotic Methods and Perturbation Theory (New York: McGraw-Hill Press)

Bunn, E. F. \& Hogg, D. W., 2009, AmJPh, 77, 688

Calder, L. \& Lahav, O., 2008, A\&G, 49, 010000

de Sitter, W., 1917, KNAB, 19, 1217

Francis, M. J., Barnes, L. A., James, J. B. \& Lewis, G. F., 2007, PASA, 24, 95

Grøn, Ø., 2006, EJPh, 27, 561

Grøn, Ø. \& Elgarøy, Ø., 2007, AmJPh, 75, 151

Heyl, J. S., 2005, PhRvD, 72, 107302

Infeld, L. \& Schild, A., 1945, PhRv, 68, 250

Krauss, L. M. \& Scherrer, R. J., 2007, GReGr, 39, 1545

Lewis, G. F., Francis, M. J., Barnes, L. A., Kwan, J. \& James, J. B., 2008, MNRAS, 388, 960

Lewis, G. F., Francis, M. J., Barnes, L. A. \& James, J. B., 2007, MNRAS, 381, L50

Loeb, A., 2002, PhRvD, 65, 047301-1

Milne, E. A., 1932, Natur, 130, 9

Perlmutter, S. et al., 1999, ApJ, 517, 565

Riess, A. G. et al., 1998, ApJ, 116, 1009

Rindler, W., 1960, PhRv, 119, 2082

Rindler, W., 2006, Relativity: Special, General, Cosmological (2nd edn) (Oxford: Oxford University Press)

Tipler, F. J., 1996, MNRAS, 282, 206

Whiting, A. B., 2004, Obs, 124, 174

\section{Appendix: Calculations}

We can solve for the first order perturbative solution to the full GR equation of motion by using the method of multiple scales [cf. Bender \& Orszag (1978)]. After performing a conformal transformation and introducing the standard relationships between the four-velocity and fouracceleration in Equations 4-6, we obtain the following differential equation for the conformal time as a function of the proper time of the rocketeer:

$$
\begin{aligned}
\frac{\mathrm{d} u^{\eta}}{\mathrm{d} \tau}= & -\frac{\mathrm{d} a(\eta)}{\mathrm{d} \eta} \frac{1}{a(\eta)} u^{\eta^{2}}+\frac{\mathrm{d} a(\eta)}{\mathrm{d} \eta} \frac{1}{a(\eta)^{3}} \\
& +\frac{\epsilon}{a(\eta)} \sqrt{a(\eta)^{2} u^{\eta^{2}}-1} .
\end{aligned}
$$

Of course the radial coordinate of the rocketeer is simply given by solving the corresponding geodesic equation in $r$ as follows:

$$
\frac{\mathrm{d} u^{r}}{\mathrm{~d} \tau}=-2 \frac{\mathrm{d} a(\eta)}{\mathrm{d} \eta} \frac{1}{a(\eta)} u^{r} u^{\eta}+\epsilon u^{\eta} .
$$

To solve these, we introduced a perturbation in $\epsilon$ and a new timescale such that:

$$
\begin{aligned}
\eta & =\eta_{0}(\tau, \bar{t})+\epsilon \eta_{1}(\tau, \bar{t})+\epsilon^{2} \eta_{2}(\tau, \bar{t})+O\left(\epsilon^{3}\right) \text { and } \\
r & =r_{0}(\tau, \bar{t})+\epsilon r_{1}(\tau, \bar{t})+\epsilon^{2} r_{2}(\tau, \bar{t})+O\left(\epsilon^{3}\right)
\end{aligned}
$$

where $\bar{t}=\epsilon \tau$, such that $\eta$ and $r$ are not truly functions of two variables. We can then write down Equations 13 and 14 as a system of PDEs. The first order solution for $u^{\eta}$ is given by:

$$
u^{\eta}=\frac{\cosh (\epsilon \tau / 2)}{a(\eta)} .
$$

Substituting this into Equation 14 then gives an integral solution for $u^{r}$ :

$$
u^{r}=\frac{\epsilon}{a^{2}(\eta)} \int a(\eta) \cosh \left(\frac{\epsilon \tau}{2}\right) \mathrm{d} \tau .
$$

For certain forms of the scale factor, we can explicitly integrate these equations again for the trajectory of the rocketeer in terms of the proper time that she measures. If we choose a power-law solution for the scale factor for simplicity, $a(t)=\left(t / t_{0}\right)^{2 / 3(1+w)}$, where $w$ is the equation of state parameter, and use Equation 3 to relate $\eta$ to $t$, we can write down $a(\eta)$ as follows:

$$
a(\eta)=\frac{1+3 w}{3 t_{0}(1+w)}\left[\eta+\frac{1+3 w}{3 t_{0}(1+w)}\right]^{2 /(1+3 w)},
$$

for $\left.a(\eta)\right|_{\eta=0}=1$. The solutions for $\eta_{0}$ and $r_{0}$ obtained from integrating $u^{\eta}$ and $u^{r}$ are:

$$
\begin{aligned}
\eta_{0}= & \frac{3(1+w)}{1+3 w}\left\{t_{0}^{2 / 3(1+w)}\right. \\
& {\left.\left[2 \sinh \left(\frac{\epsilon \tau}{2}\right)+t_{0}\right]^{(1+3 w) / 3(1+w)}-t_{0}\right\}, } \\
r_{0}= & a t_{0} \frac{3(1+w)}{5+3 w} \int_{0}^{\tau}\left[f(\bar{\tau})^{(1+3 w) / 3(1+w)}\right. \\
& \left.-f(\bar{\tau})^{-4 / 3(1+w)}\right] \mathrm{d} \bar{\tau},
\end{aligned}
$$

where $f(\tau)=\left(2 / \epsilon t_{0}\right) \sinh (\epsilon \tau / 2)+1$. Note that it is not correct to directly substitute $\eta_{0}$ into the equation for the normalisation of the four-velocity to derive an expression for $u_{r}$, because the first-order solutions do not necessarily satisfy these conditions exactly, so the result obtained is not strictly a first order perturbation.

It is important to remember that these are first order solutions and hence at most accurate to $\tau=O(1 / \epsilon)$. The accuracy of these solutions is demonstrated in Figure 7 , in which we compare them to numerical results and the solution in special relativity. The difference between the solutions contained in Figure 7 and the numerical result is smaller than the same comparison for special relativity. Thus, if we were looking for a way to approximate the full behaviour in general relativity, the use of perturbative methods on the geodesic equation is preferable to using special relativity for even moderately distant destinations, in this instance, anything further than $0.4 H_{0}^{-1}$. 